\title{
Magnitude of beam-hardening artifacts produced by gutta-percha and metal posts on cone- beam computed tomography with varying tube current
}

\author{
Hugo Gaêta-Araujo $\mathbb{D}^{1}$, Eduarda Helena Leandro Nascimento $\mathbb{D}^{1}$, Rocharles Cavalcante Fontenele $\mathbb{D}^{1}$, \\ Arthur Xavier Maseti Mancini $\mathbb{D}^{2}$, Deborah Queiroz Freitas $\mathbb{D}^{1}$, Christiano Oliveira-Santos $\mathbb{D}^{3, *}$ \\ ${ }^{1}$ Department of Oral Diagnosis, Division of Oral Radiology, Piracicaba Dental School, University of Campinas, Piracicaba, Sao Paulo, Brazil \\ ${ }^{2}$ Department of Dental Materials and Prosthodontics, School of Dentistry of Ribeirao Preto, University of Sao Paulo, Ribeirao Preto, \\ Sao Paulo, Brazil \\ ${ }^{3}$ Department of Stomatology, Public Oral Health and Forensic Dentistry, Division of Oral Radiology, School of Dentistry of Ribeirao \\ Preto, University of Sao Paulo, Ribeirao Preto, Sao Paulo, Brazil
}

\section{ABSTRACT}

Purpose: This study was performed to evaluate the magnitude of artifacts produced by gutta-percha and metal posts on cone-beam computed tomography (CBCT) scans obtained with different tube currents and with or without metal artifact reduction(MAR).

Materials and Methods: A tooth was inserted in a dry human mandible socket, and CBCT scans were acquired after root canal instrumentation, root canal filling, and metal post placement with various tube currents with and without MAR activation. The artifact magnitude was assessed by the standard deviation (SD) of gray values and the contrastto-noise ratio $(\mathrm{CNR})$ at the various distances from the tooth. Data were compared using multi-way analysis of variance. Results: At all distances, a current of $4 \mathrm{~mA}$ was associated with a higher SD and a lower CNR than $8 \mathrm{~mA}$ or $10 \mathrm{~mA}$ $(P<0.05)$. For the metal posts without MAR, the artifact magnitude as assessed by SD was greatest at $1.5 \mathrm{~cm}$ or less $(P<0.05)$. When MAR was applied, SD values for distances $1.5 \mathrm{~cm}$ or closer to the tooth were reduced $(P<0.05)$. MAR usage did not influence the magnitude of artifacts in the control and gutta-percha groups $(P>0.05)$.

Conclusion: Increasing the tube current from $4 \mathrm{~mA}$ to $8 \mathrm{~mA}$ may reduce the magnitude of artifacts from metal posts. The magnitude of artifacts arising from metal posts was significantly higher at distances of $1.5 \mathrm{~cm}$ or less than at greater distances. MAR usage improved image quality near the metal post, but had no significant influence farther than $1.5 \mathrm{~cm}$ from the tooth.(Imaging Sci Dent 2020; 50: 1-7)

KEY WORDS: Cone-Beam Computed Tomography; Artifacts; Gutta-Percha; Metals

\section{Introduction}

Artifacts impair the image quality of cone-beam computed tomography (CBCT) scans and may hinder the diagnosis of numerous conditions. Among the types of artifacts on CBCT scans, those derived from the beam-hardening

This study was financed in part by the Coordenação de Aperfeiçoamento de Pessoal de Nível Superior - Brasil (CAPES) - Finance Code 001.

Received July 30, 2019; Revised September 26, 2019; Accepted October 10, 2019 *Correspondence to : Prof. Christiano de Oliveira-Santos

Department of Stomatology, Public Oral Health and Forensic Dentistry, Division of Oral Radiology, School of Dentistry of Ribeirao Preto, University of Sao Paulo, Av. do Café, s/n, Vila Monte Alegre, Zip Code 14040-904, Ribeirão Preto, SP, Brazil Tel) 55-16-3315-3976, E-mail) oliveirach@usp.br effect may critically impact diagnosis and are caused by high-density materials located inside ${ }^{1,2}$ or outside ${ }^{3}$ the field of view. Such materials include dental restorations and metal crowns, dental implants, gutta-percha, and metal posts. Restorations and crowns create artifacts at the level of dental crowns, which may affect the diagnosis of carious lesions, ${ }^{4}$ while the other materials generate artifacts at the level of dental roots and alveolar bone, negatively influencing the ability of clinicians to detect conditions such as root fractures, ${ }^{5,6}$ root resorptions, ${ }^{7}$ and bone defects. ${ }^{8}$

The production of artifacts in regions adjacent to highdensity materials is relatively well understood. ${ }^{9,10}$ However, recent reports have begun to address the spread of arti- 
facts to different regions of the CBCT volume. ${ }^{1,2}$ Further investigation of this possibility is important for confirming whether errors in the reconstructed data can affect the image quality in regions distant from the artifact-generating material.

Although the presence of metal posts and gutta-percha is very common in dental examinations, until now, studies investigating the magnitude of the artifacts in CBCT images have only focused on dental implants. ${ }^{1,2,6,7}$ As different materials produce artifacts of different magnitude and as endodontic materials typically have a high atomic number, ${ }^{11}$ studying how the artifacts relate to these materials throughout the volume of the CBCT scan is relevant. Since the tube current is directly related to the administered radiation dose, it should be set as low as diagnostically acceptable. ${ }^{12-14}$ As the current is increased, image noise is expected to decrease, but no clear relationship with the presence of beam-hardening artifacts has been found. ${ }^{15,16}$ Metal artifact reduction (MAR) tools are post-processing algorithms developed by the manufacturers of some CBCT devices to improve image quality during the reconstruction process by reducing beam-hardening artifacts. ${ }^{10}$ However, information on how such algorithms influence the image is limited.

As such, the aim of this study was to evaluate the magnitude of artifacts produced due to the beam-hardening phenomenon by gutta-percha and metal posts in CBCT scans obtained with different tube currents and with or without the usage of MAR.

\section{Materials and Methods}

This study was approved by the local institutional review board (protocol no. 07908919.7.0000.5418). The study sample was composed of a partially dentate dry human mandible and an extracted single-rooted tooth.

\section{Sample preparation}

The tooth was disinfected with $70 \%$ alcohol, was scaled to remove calculus and soft tissue remnants, and had its crown sectioned along the cemento-enamel junction with a diamond saw (Isomet 1000, Buehler Ltd., Lake Bluff, IL, USA). A root canal instrumentation was implemented according to the MTwo (VDW GmbH, Munich, Germany) protocol (size/taper: 10/0.04, 15/0.05, 20/0.06, and 25/0.06) with distilled water irrigation between rotatory files. The root canal was filled along its entire length using gutta-percha and the endodontic cement sealer AH Plus (Dentsply Sirona Endodontics, Charlotte, NC, USA) according to the lateral condensation technique.
The root canal was subsequently prepared for metal post placement by removal of the root filling from the initial two-thirds of the root using \#2 and \#3 piezo drills (Peeso Long Drill, Dentsply Sirona Endodontics). The metal post was molded using a direct technique with a standard dowel made of Duralay acrylic resin. A nickel-chromium (NiCr) post was cast using a FIT CAST-SB Plus alloy (Talmax Ltda, Curitiba, Brazil). Metal post fitting was observed by visual inspection and periapical radiography.

\section{CBCT acquisition}

Scans were acquired for each intracanal condition: after root canal instrumentation without filling, 1 day after root canal filling, and after metal post placement. For CBCT scan acquisition, the right second premolar socket of the dry mandible was enlarged to fit the tooth passively. A block of homogeneous condensation silicone impression material (Precise SX, Dentsply Sirona, Sao Paulo, Brazil) was attached to the buccal cortical region of the mandible to allow the selection of the same slice on the contralateral side to serve as a control area for subsequent image analysis. The mandible was inserted into a plastic receptacle and filled with water to simulate soft tissue attenuation of the X-ray beam. This set was positioned in an OP300 Maxio unit (Instrumentarium Dental, Tuusula, Finland) with the occlusal plane parallel to the horizontal plane. The acquisition protocol consisted of a field of view of $6 \mathrm{~cm} \times 8 \mathrm{~cm}$, a peak kilovoltage of $90 \mathrm{kVp}$, and a voxel size of $0.2 \mathrm{~mm}$, with a total scan time of $17.4 \mathrm{~s}$ and an exposure time of $6.09 \mathrm{~s}$. Tube current was set at 3 levels (4 $\mathrm{mA}, 8 \mathrm{~mA}$, and $10 \mathrm{~mA}$ ) either with or without activation of the MAR tool. As such, a total of 6 protocols were used for each intracanal canal condition: $4 \mathrm{~mA}$ without MAR, 4 $\mathrm{mA}$ with MAR, $8 \mathrm{~mA}$ without MAR, $8 \mathrm{~mA}$ with MAR, 10 $\mathrm{mA}$ without MAR, and $10 \mathrm{~mA}$ with MAR. Each protocol was performed 3 times for each intracanal condition, totaling 54 acquisitions ( 3 intracanal conditions $\times 3 \mathrm{~mA}$ levels $\times 2$ MAR conditions $\times 3$ repetitions $)$.

\section{Image assessment}

For each volume, an oral and maxillofacial radiologist used OnDemand3D software version 1.0 (Cybermed Inc., Seoul, Korea) to select the axial image corresponding to the upper level of the block of homogeneous material positioned in the buccal cortical plate close to the artifact-generating material.

All axial images were exported as Digital Imaging and Communications in Medicine files and were opened with ImageJ software version 1.51 (National Institutes of Health, 


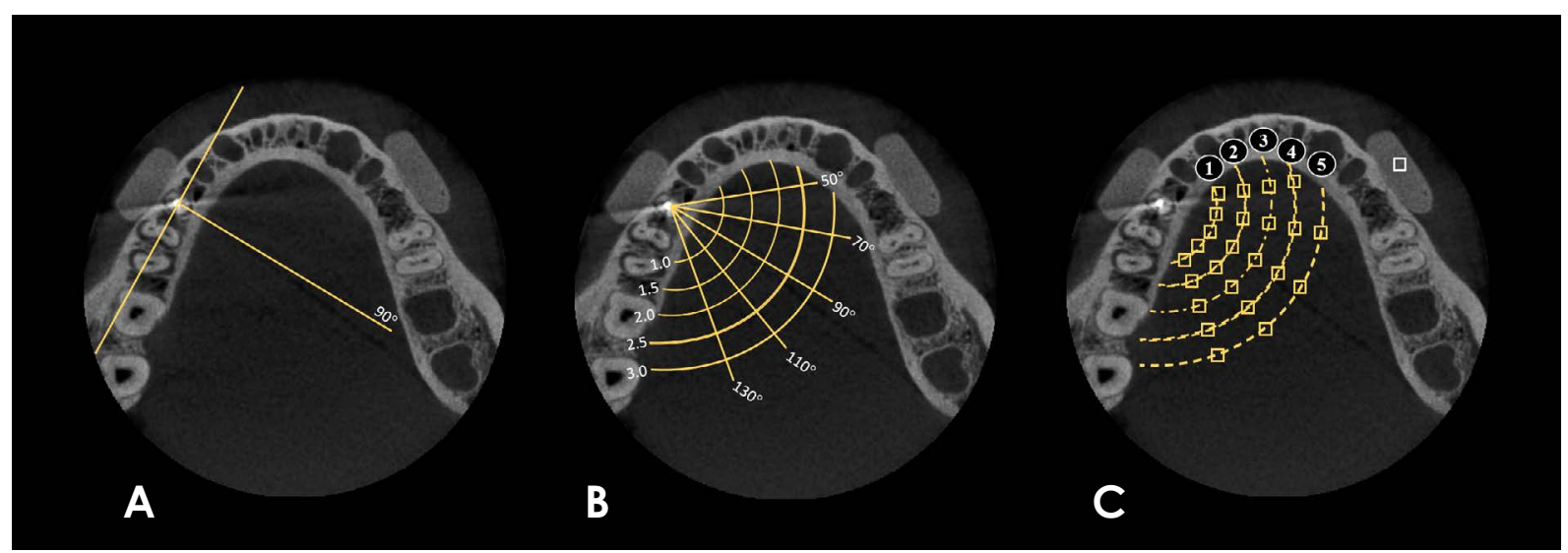

Fig. 1. A. A line was drawn perpendicular to the mandibular body and through the center of the artifact-generating object. B. $20^{\circ}$-step lines were drawn above and below the perpendicular line, and 5 concentric semicircles were created with different radii (0.5-cm intervals). C. In the regions of intersection between the lines and the semicircles, 24 square regions of interest (ROIs) of the same size were established. A square ROI of the same size as the others was established in the silicone block, serving as the control area.

Bethesda, MD, USA). In each axial image, 24 square regions of interest (ROIs) of the same size $(2 \mathrm{~mm} \times 2 \mathrm{~mm})$ were established at different positions and distances from the root, using lines and semicircles as references (Fig. 1). Initially, a line was drawn parallel to the long axis of the mandible body on the right side and passing through the center of the artifact-generating object. A second line was drawn perpendicular to the first line (Fig. 1A). Four additional lines were then drawn at different angles to the perpendicular line: 2 anterior $\left(50^{\circ}\right.$ and $\left.70^{\circ}\right)$ and 2 posterior $\left(110^{\circ}\right.$ and $\left.130^{\circ}\right)$ (Fig. 1B). After establishing these lines, 5 semicircles centered on the artifact-generating object were drawn, starting with a radius of $1.0 \mathrm{~cm}$ and increasing by consecutive $0.5-\mathrm{cm}$ increments. The ROIs were established in the areas of intersection between the lines and the semicircles (Fig. 1C).

For the control area, an additional ROI with the same shape and size was selected from the silicone block located in the buccal cortical plate of the left mandible body. This ROI was essential in the calculation of the contrast-noise ratio (CNR) according to the equation described by Bechara et al. in 2012: ${ }^{17}$

$$
C N R=\frac{\mid \text { Mean }_{R O I}-\text { Mean }_{\text {Control }} \mid}{\sqrt{S D_{R O I}^{2}+S D_{\text {Control }}^{2}}}
$$

For each ROI, the mean standard deviation (SD) of the gray values was obtained. The ROI position was standardized using the ROI manager tool within the ImageJ software. All analyses were performed using 8-bit images. The values obtained from the 24 ROIs were grouped into 5 ra- dial distances $(1 \mathrm{~cm}, 1.5 \mathrm{~cm}, 2.0 \mathrm{~cm}, 2.5 \mathrm{~cm}$, and $3.0 \mathrm{~cm})$ in order to express the magnitudes of the generated artifacts and the effects of the factors studied (Fig. 1C).

\section{Statistical analysis}

The analysis was performed using IBM SPSS Statistics software version 24.0 (IBM Corp., Armonk, NY, USA) and GraphPad Prism version 7.0 (GraphPad Software, La Jolla, CA, USA), with the significance level set at $P<0.05$.

The SD and CNR values were compared using multiway analysis of variance with the Tukey post-hoc test in order to test the main effects of artifact-generating object type, tube current, MAR tool usage (Fig. 2) and distance. The null hypothesis was that the factors studied would have no influence on SD or CNR values.

\section{Results}

Table 1 shows the mean SDs obtained at each distance for each intracanal material with varying current and with or without the activation of the MAR tool. Regardless of distance, intracanal material, and MAR usage, a current of $4 \mathrm{~mA}$ was associated with a higher $\mathrm{SD}(P<0.05)$. For the metal post group without MAR use, distances of $1 \mathrm{~cm}$ and $1.5 \mathrm{~cm}$ were associated with significantly greater artifact expression than larger distances $(P<0.05)$. When MAR was activated, the values for the distances of $1 \mathrm{~cm}$ and 1.5 $\mathrm{cm}$ were reduced in the metal post group $(P<0.05)$ and did not significantly differ from those for the other distances $(P>0.05)$. MAR usage did not significantly influence artifact expression at any distance for the control and gut- 


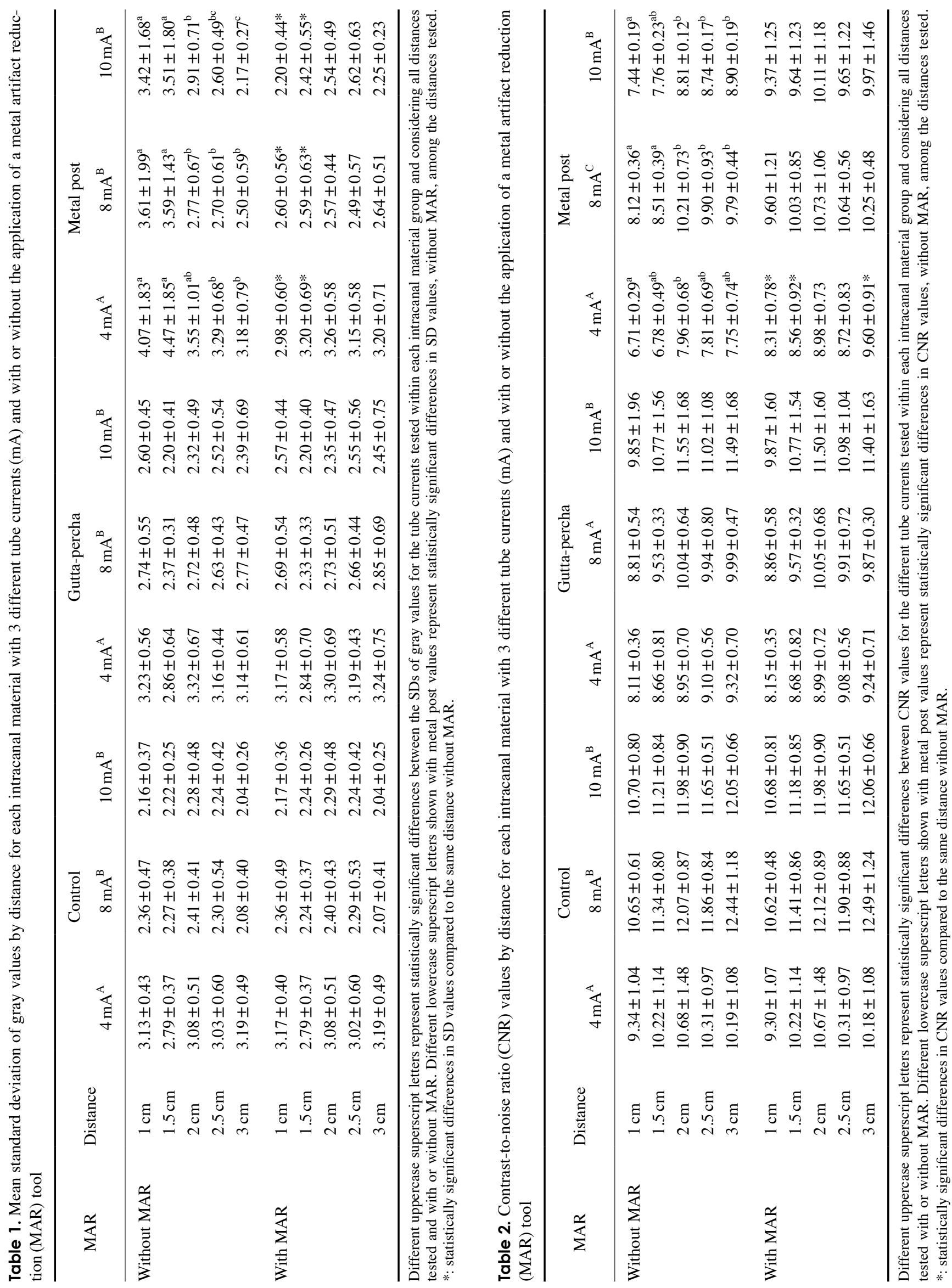




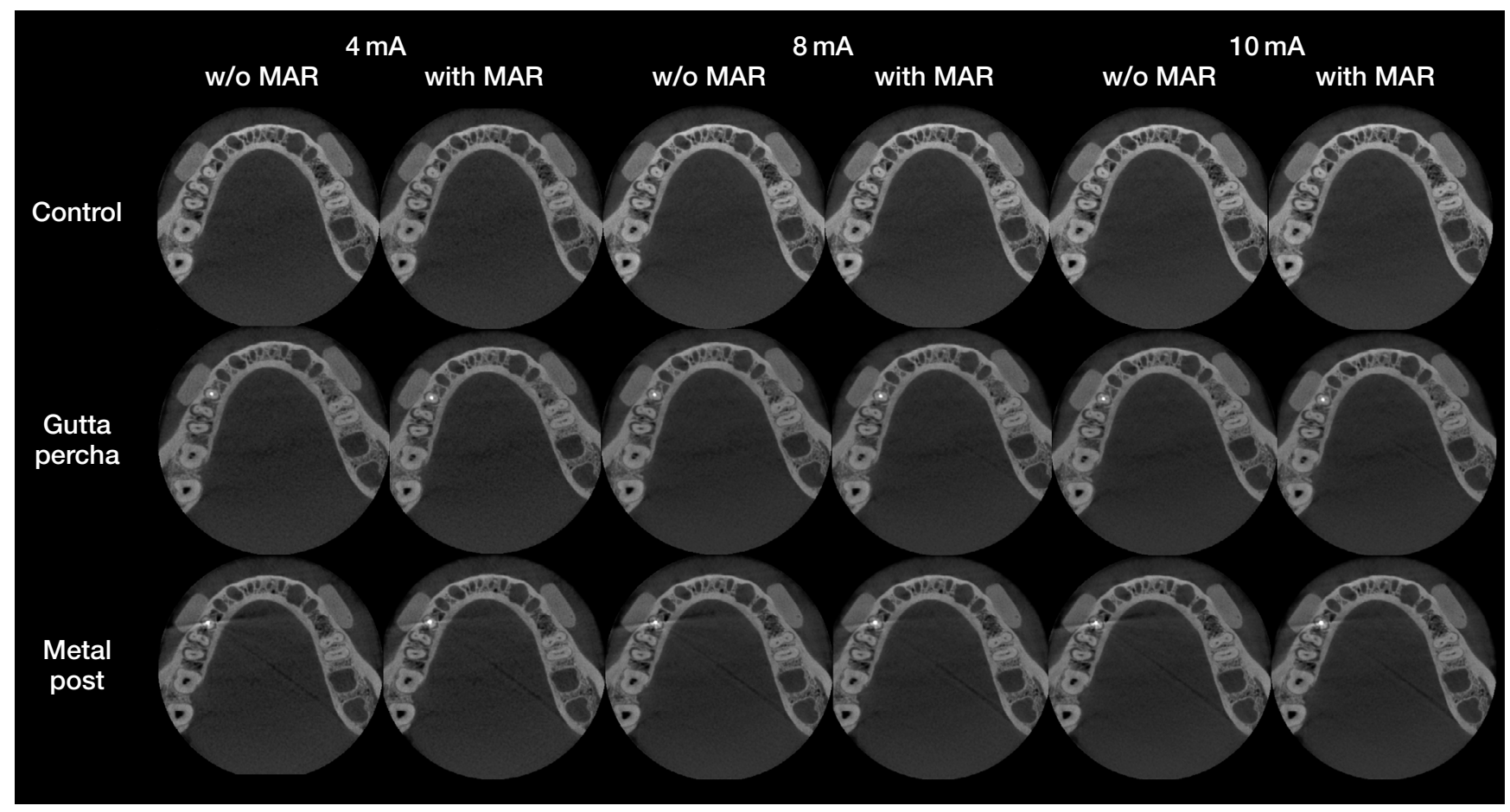

Fig. 2. Axial images represent intracanal materials (control in the first row, gutta-percha in the second row, and metal post in the third row) with different tube currents $(4 \mathrm{~mA}, 8 \mathrm{~mA}$, and $10 \mathrm{~mA})$ and without and with the application of a metal artifact reduction tool.

ta-percha groups $(P>0.05)$.

Mean CNR values are shown in Table 2. For the control and gutta-percha groups, no significant differences were observed according to distance and MAR activation $(P>0.05)$. However, a current of $4 \mathrm{~mA}$ was associated with lower CNR values than either $8 \mathrm{~mA}$ or $10 \mathrm{~mA}$ in the control group and $10 \mathrm{~mA}$ in the gutta-percha group $(P<0.05)$. For the metal post group without MAR, all tube currents showed significant differences, with the lowest CNR occurring at $4 \mathrm{~mA}$ and the highest at $8 \mathrm{~mA}(P<0.05)$. In that group (metal post without MAR), at the tube currents of $8 \mathrm{~mA}$ and $10 \mathrm{~mA}, \mathrm{CNR}$ values were significantly higher for ROIs located at larger distances. MAR activation only yielded a significant increase in the CNR in the metal post group with a current of $4 \mathrm{~mA}$ at the distances of $1 \mathrm{~cm}, 1.5$ $\mathrm{cm}$, and $3 \mathrm{~cm}(P<0.05)$.

\section{Discussion}

In the present study, the magnitude of artifacts arising from gutta-percha and metal posts was evaluated. Artifacts arising from metal posts were significantly higher in magnitude at distances of $1.5 \mathrm{~cm}$ or less from the tooth. This may have a relevant clinical impact in the assessment of anatomical structures in the vicinity of teeth restored with metal posts in CBCT scans taken for various diagnostic purposes. A previous study ${ }^{1}$ assessed the magnitude of artifacts originating from titanium and zirconium dental implants and found artifacts from zirconium implants as far as $3.5 \mathrm{~cm}$ from the tooth. However, for titanium, the expression of artifacts occurred relatively close to the implant, as observed for metal posts in the present study. The metal alloy used for the metal post cast in this study was $\mathrm{Ni}-\mathrm{Cr}$, the components of which have similar atomic numbers as titanium (Ni, $Z=28 ; \mathrm{Cr}, \mathrm{Z}=24 ; \mathrm{Ti}, \mathrm{Z}=22)$. This fact may explain the similar behavior regarding artifact magnitude.

The finding that the use of gutta-percha had little effect on the magnitude of artifacts may relate to its composition. Although one of the components of gutta-percha is zinc oxide $(\mathrm{Z}=30)$, and zinc has a greater atomic number than $\mathrm{Ni}$ or $\mathrm{Cr}$, there are also organic components in gutta-percha (resin and wax), with extremely low atomic numbers. ${ }^{10}$ Additionally, zinc oxide is less physically dense $\left(\mathrm{d}=5.1 \mathrm{~g} / \mathrm{cm}^{3}\right)$ than both nickel $\left(\mathrm{d}=8.9 \mathrm{~g} / \mathrm{cm}^{3}\right)$ and chromium $\left(\mathrm{d}=7.2 \mathrm{~g} / \mathrm{cm}^{3}\right)$.

The 3 variables analyzed in the present study (tube current, artifact-producing material, and MAR tool usage) can affect CBCT image quality. They are related as follows: lower tube currents and/or the presence of high-density materials tend to produce images with higher SD values and 
lower CNRs; ${ }^{1}$ in contrast, the usage of a MAR tool reduces the variability of image gray values, ${ }^{2,10}$ acting mainly in areas where artifacts are more highly expressed (areas with high SD and low CNR). When beam-hardening artifacts are not present, however, MAR tool usage does not change the gray values of the image. ${ }^{18}$

The acquisition parameters of CBCT affect both the magnitude of artifacts and the dose to which the patient is exposed. As shown previously, increasing the peak kilovoltage can reduce artifact magnitude. ${ }^{2}$ However, the relationship between image quality and dose at various peak kilovoltages is not straightforward. ${ }^{12}$ As far as we know, this is the first study to assess the influence of tube current on artifact magnitude. Recently, a study assessed the impact of tube current on metal post artifact formation in the context of dental structures alone and concluded that tube current did not affect artifact intensity. ${ }^{11}$ Conversely, in the present study, for every intracanal condition, a tube current of $4 \mathrm{~mA}$ was associated with higher artifact formation (higher SD and lower CNR) than currents of $8 \mathrm{~mA}$ and 10 $\mathrm{mA}$, even when no artifact-generating material was present. The difference in the ROI location evaluated in these studies may explain the divergent results. Additionally, the previous study did not use currents as low as $4 \mathrm{~mA}$; instead, only currents of $6.3 \mathrm{~mA}$ and $10 \mathrm{~mA}$ were evaluated.

In this study, image noise decreased with increased tube current, as expected. The differences in SD between tube currents observed in this study may also be attributed to better image quality with lower noise. CNR values were similarly affected by current, showing that an increase in tube current from $4 \mathrm{~mA}$ to $8 \mathrm{~mA}$ was capable of decreasing the magnitude of artifacts from metal posts.

For gutta-percha, which was associated with lower artifact expression than metal posts, the effect on image quality was only significant when tube current was increased to $10 \mathrm{~mA}$. However, considering that the impact of gutta-percha on image quality is already much more limited than that of metal posts and that tube current must be set as low as possible due to its direct relationship with the radiation dose, it is questionable whether increasing the tube current in such cases should be recommended.

It is important to emphasize that the application of MAR in CBCT imaging is a post-processing step and therefore does not influence the radiation dose delivered to the patient, although it does increase the reconstruction time. ${ }^{10}$ The use of MAR was effective only for the metal post in the regions closer to the root, leading to similar SDs among the regions at every tube current, but only leading to similar CNR values at a current of $4 \mathrm{~mA}$. The MAR tool has also been found to be effective in the homogenization of gray values in dental implant artifacts. ${ }^{2}$ Indeed, MAR acts when pronounced artifacts are present in the image; ${ }^{2,10}$ therefore, MAR was not expected to influence the control and gutta-percha groups. However, there is no consensus in the literature regarding the influence of MAR, as it does not correct the gray values in the vicinity of dental implants, ${ }^{19}$ and its use has not been found to improve the diagnostic accuracy of certain tasks. ${ }^{8,20,21}$ Further studies should be carried out to assess the true impact of MAR under different circumstances.

Because a variety of materials are applied in dentistry, information regarding the resulting artifacts must be acknowledged to minimize the impact of these artifacts on diagnostic tasks and to optimize image acquisition. The metal alloy chosen for the present study was $\mathrm{Ni}-\mathrm{Cr}$, which has been shown to produce artifact formation similar to a silver-palladium alloy when alone in the dental arch. ${ }^{11}$ Additionally, CBCT imaging with more than 1 artifact-generating object in the field of view has been shown to be jeopardized by greater artifact expression due to photon starvation. ${ }^{11,16,22}$ Therefore, the magnitude of artifacts from different alloys and the quantity in the dental arch should be assessed in future studies.

In conclusion, an increase in tube current from $4 \mathrm{~mA}$ to $8 \mathrm{~mA}$ may reduce noise and metal post-related artifacts. The magnitude of artifacts arising from metal posts was significantly higher at distances shorter than $1.5 \mathrm{~cm}$ from the tooth. Beyond that point, the magnitude decreased significantly. MAR usage improved image quality in regions close to the metal post; however, it did not significantly influence image quality at distances farther than $1.5 \mathrm{~cm}$ from the tooth.

\section{Conflicts of Interest: None}

\section{References}

1. Fontenele RC, Nascimento EH, Vasconcelos TV, Noujeim M, Freitas DQ. Magnitude of cone beam CT image artifacts related to zirconium and titanium implants: impact on image quality. Dentomaxillofac Radiol 2018; 47: 20180021.

2. Freitas DQ, Fontenele RC, Nascimento EH, Vasconcelos TV, Noujeim M. Influence of acquisition parameters on the magnitude of cone beam computed tomography artifacts. Dentomaxillofac Radiol 2018; 47: 20180151.

3. Candemil AP, Salmon B, Freitas DQ, Ambrosano GM, Haiter-Neto F, Oliveira ML. Metallic materials in the exomass impair cone beam CT voxel values. Dentomaxillofac Radiol 2018; 47: 20180011. 
4. Cebe F, Aktan AM, Ozsevik AS, Ciftci ME, Surmelioglu HD. The effects of different restorative materials on the detection of approximal caries in cone-beam computed tomography scans with and without metal artifact reduction mode. Oral Surg Oral Med Oral Pathol Oral Radiol 2017; 123: 392-400.

5. Neves FS, Freitas DQ, Campos PS, Ekestubbe A, Lofthag-Hansen $\mathrm{S}$. Evaluation of cone-beam computed tomography in the diagnosis of vertical root fractures: the influence of imaging modes and root canal materials. J Endod 2014; 40: 1530-6.

6. Freitas DQ, Vasconcelos TV, Noujeim M. Diagnosis of vertical root fracture in teeth close and distant to implant: an in vitro study to assess the influence of artifacts produced in cone beam computed tomography. Clin Oral Investig 2019; 23: 1263-70.

7. Freitas DQ, Nascimento EH, Vasconcelos TV, Noujeim M. Diagnosis of external root resorption in teeth close and distant to zirconium implants: influence of acquisition parameters and artefacts produced during cone beam computed tomography. Int Endod J 2019; 52: 866-73.

8. de-Azevedo-Vaz SL, Peyneau PD, Ramirez-Sotelo LR, Vasconcelos Kde F, Campos PS, Haiter-Neto F. Efficacy of a cone beam computed tomography metal artifact reduction algorithm for the detection of peri-implant fenestrations and dehiscences. Oral Surg Oral Med Oral Pathol Oral Radiol 2016; 121: 550-6.

9. Sancho-Puchades M, Hämmerle $\mathrm{CH}$, Benic GI. In vitro assessment of artifacts induced by titanium, titanium-zirconium and zirconium dioxide implants in cone-beam computed tomography. Clin Oral Implants Res 2015; 26: 1222-8.

10. Queiroz PM, Oliveira ML, Groppo FC, Haiter-Neto F, Freitas DQ. Evaluation of metal artefact reduction in cone-beam computed tomography images of different dental materials. Clin Oral Investig 2018; 22: 419-23.

11. Lira de Farias Freitas AP, Cavalcanti YW, Costa FC, Peixoto LR, Maia AM, Rovaris K, et al. Assessment of artefacts produced by metal posts on CBCT images. Int Endod J 2018; 52 : 223-36.

12. Pauwels R, Silkosessak O, Jacobs R, Bogaerts R, Bosmans H, Panmekiate S. A pragmatic approach to determine the optimal $\mathrm{kVp}$ in cone beam CT: balancing contrast-to-noise ratio and radiation dose. Dentomaxillofac Radiol 2014; 43: 20140059.

13. Jaju PP, Jaju SP. Cone-beam computed tomography: time to move from ALARA to ALADA. Imaging Sci Dent 2015; 45 : 263-5.

14. Pauwels R, Seynaeve L, Henriques JC, de Oliveira-Santos C, Souza PC, Westphalen FH, et al. Optimization of dental CBCT exposures through mAs reduction. Dentomaxillofac Radiol 2015; 44: 20150108.

15. Schulze R, Heil U, Gross D, Bruellmann DD, Dranischnikow E, Schwanecke U, et al. Artefacts in CBCT: a review. Dentomaxillofac Radiol 2011; 40: 265-73.

16. Pauwels R, Stamatakis H, Bosmans H, Bogaerts R, Jacobs R, Horner K, et al. Quantification of metal artifacts on cone beam computed tomography images. Clin Oral Implants Res 2013; 24 Suppl A100: 94-9.

17. Bechara B, McMahan CA, Geha H, Noujeim M. Evaluation of a cone beam CT artefact reduction algorithm. Dentomaxillofac Radiol 2012; 41: 422-8.

18. Nascimento EH, Fontenele RC, Santaella GM, Freitas DQ. Difference in the artefacts production and the performance of the metal artefact reduction (MAR) tool between the buccal and lingual cortical plates adjacent to zirconium dental implant. Dentomaxillofac Radiol 2019; 48: 20190058.

19. Parsa A, Ibrahim N, Hassan B, Syriopoulos K, van der Stelt P. Assessment of metal artefact reduction around dental titanium implants in cone beam CT. Dentomaxillofac Radiol 2014; 43 : 20140019.

20. Kamburoğlu K, Yilmaz F, Yeta EN, Özen D. Assessment of furcal perforations in the vicinity of different root canal sealers using a cone beam computed tomography system with and without the application of artifact reduction mode: an ex vivo investigation on extracted human teeth. Oral Surg Oral Med Oral Pathol Oral Radiol 2016; 121: 657-65.

21. Nikbin A, Dalili Kajan Z, Taramsari M, Khosravifard N. Effect of object position in the field of view and application of a metal artifact reduction algorithm on the detection of vertical root fractures on cone-beam computed tomography scans: an in vitro study. Imaging Sci Dent 2018; 48: 245-54.

22. Vasconcelos KF, Codari M, Queiroz PM, Nicolielo LF, Freitas DQ, Sforza C, et al. The performance of metal artifact reduction algorithms in cone beam computed tomography images considering the effects of materials, metal positions, and fields of view. Oral Surg Oral Med Oral Pathol Oral Radiol 2019; 127: 71-6. 